\title{
A Case of Anti TNF Induced Leukopenia Responding to Small Dose of Prednisolone in Patient with Ankylosing Spondylitis
}

\author{
Hanan A Alhamzi ${ }^{1 *}$, Osamah A Alkhaled ${ }^{2}$, Mosaab A Makkawy ${ }^{1}$ and Rowad Nori ${ }^{1}$
}

${ }^{1}$ Adult Rheumatology, Rheumatology Consultant, Prince Mohammed bin Abdulaziz Hospital

${ }^{2}$ Internal Medicine, Prince Mohammad Bin Abdulaziz Hospital, Riyadh, Saudia Arabia

\begin{abstract}
Received: August 13, 2017; Accepted: October 28, 2017; Published: November 06, 2017
*Corresponding author: Hanan A Alhamzi, Rheumatology Consultant, Prince Mohammed bin Abdulaziz Hospital, Saudi Arabia, E-mail: alhamzih@ yahoo.com
\end{abstract}

\begin{abstract}
Anti Tumor Necrosis Factor (TNF) is an important therapy for many rheumatological diseases, we report 37 years old male patient with ankylosing spondylitis who developed anti-TNF induced neutropenia that responded well to small dose of prednisolone. We discussed the possible mechanism of anti-TNF induced neutropenia.
\end{abstract}

Keywords: Anti tumor necrosis factor; Neutropeni; Prednisolone;

\section{Introduction}

Anti TNF therapy plays a major role in treatment of patient with ankylosing spondylitis. Hematological complications are rare and there is no recommendation in the guideline to do regular full blood count after initiation of anti TNF. However, several studies and case reports showed that leukopenia induced by anti TNF can happen in up to $18.8 \%$ of cases [1].

Though the leukopenia induced by anti TNF is reversible upon stopping medication, it will be difficult to stop anti TNF in patient with active disease.

Based on our review, this is the first case reported the benefit of adding small dose of prednisolone for patient with Anti TNF induce leukopenia to improve absolute neutrophil count .

\section{Case Report}

37 years old male patient with ankylosing spondylitis, for the last 15years, the first time was seen in rheumatology clinic.

The first time was seen in rheumatology clinic was on December 2015. He had an inflammatory back pain that interfering with his daily activities. The pain was not responding to non-steroidal anti-inflammatory drugs. MRI sacroiliac showed features of acute on top of chronic bilateral sacroiliitis. During work up his purified protein derivative skin test was found to be positive, Isoniazid $300 \mathrm{mg}$ po daily with pyridoxine were started. One month later, the patient was started on adalimumab. At that time his white blood cell count (WBC) was $6.4 \times 10^{\wedge} 9 /$ L with Absolute Neutrophilic Count (ANC): 3.2 of X10^9/ L. He has a good response in the form of no pain and improvement of the function. After one month of starting adalimumab, his WBC started to decrease

Gradually At the 11th week of starting adalimumab, his WBC became 2.9 X10 ^ $9 /$ L (ANC: 0.69 X10^9/ L) .Anti-nuclear antibody was negative. Adalimumab was stopped with regular follow up of his ANC. The ANC gradually improved to reach to $1.25 \mathrm{X} 10^{\wedge} 9 / \mathrm{L}$ over 2 months, at that time Isoniazid was stopped by infectious disease team. Later on patient restarted to complain of back pain, so etanercept was started. The follow up CBC, showed that ANC decreased gradually again after one month of starting etanercept to reach to $0.98 \mathrm{X} 10^{\wedge} \mathrm{g} / \mathrm{L}$, at that time prednisolone $5 \mathrm{mg}$ po daily was added. Follow up CBC showed that ANC went back to $2.7 \mathrm{X} 10^{\wedge} 9 / \mathrm{L}$, then prednisolone dose was decreased to $2.5 \mathrm{mg}$ po daily. See (figure 1 )

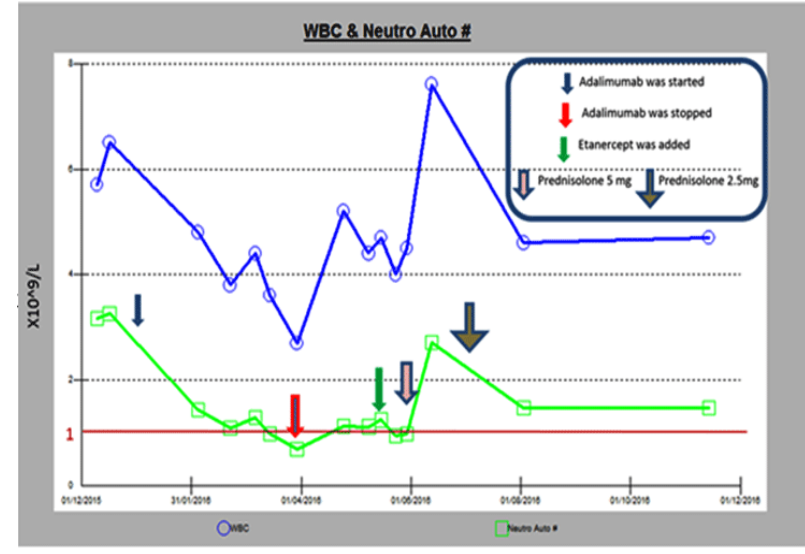

Figure 1:The association between the number of WBC/ANC and the treatment. 
After one month his WBC: $4.6 \mathrm{X} 10^{\wedge} \mathrm{9} / \mathrm{L}$ and ANC: $1.48 \mathrm{X} 10$ $\wedge 9 / \mathrm{L}$ which stay the same with regular follow up over 6 months on the same dose of prednisolone.

\section{Discussion}

Anti TNF therapy has a tremendous effect on the management of some connective tissue disease. Our patient has been treated with non-steroidal inflammatory drug for 15 years in spite of that the disease has significantly progressed.

Neutropenia have been reported with several anti TNF therapies and different autoimmune disease [2-6].

In one review of 15 articles, a total of 111 patients (receiving TNF-alpha inhibitor) with neutropenia have been reported in these studies.

Most cases were transient neutropenia, but significant severe neutropenia also developed in several patients [7].

It has been shown that the risk for anti-TNF induced leukopenia will be higher in patient who significantly had lower baseline neutrophil count $(4,2 * 10$ (9) /liter) and previous history of neutropenia while receiving disease modifying anti rheumatic [1].

However our patient's baseline WBC count was not low and was not on DMARD. His WBC was also normal after one month of isoniazid treatment onset. In addition to that his ANC decreased after starting etanercept though at that time he was not on isoniazid. The time period between starting anti-TNF treatment and developing neutropenia ranged from 1 week to 26 months, with a median of 3 months. The usual approach in anti TNF induced leukopenia has been reported to do temporary cessation, with reinstatement once the neutrophil count has recovered [8].

One of the possible mechanism that can explain the leukopenia in this case is Anti-TNF drug induced lupus, but the patient has negative Anti-Nuclear Antibody.

Another possible mechanism that had been reported in one case study is the presence of specific granulocyte auto antibodies [9].

The mechanism of anti TNF induced leukopenia is not clear yet. However, the following mechanisms are possible explanations for what happened in our patient:

1-Developing specific granulocyte auto antibodies after starting anti TNF therapy. Unfortunately we don't have the facility to test for that anti bodies.
2-Anti TNF induced Lupus is less likely as our patient has negative ANA

3-It might be related to direct inhibition of TNF action on neutrophil count but this is not supported by the patient's white cell count improvement with steroid.

Though, more studies are needed to be conducted to clarify that mechanism.

\section{Conclusion}

Prednisolone can be used in small doses to manage the anti TNF induced neutropenia.

\section{References}

1. Hastings R, Ding $\mathrm{T}$, Butt $\mathrm{S}$, Gadsby $\mathrm{K}$, Zangh $\mathrm{W}$, Moots $\mathrm{R}$, et al. Neutropenia in patients receiving anti-tumor necrosis factor therapy. Arthritis care \& research. 2010;62(6):764-769. doi: 10.1002/ acr.20037

2. Datta K, Ghosh RK, Ghosh SM. Serious neutropenia following etanercept administration in a 62 years female patient of rheumatoid arthritis. The Journal of the Association of Physicians of India. 2010;58:643-644.

3. Jordan J, Bieber T, Wilsmann-Theis D. Rapid remission of psoriasis with reversible leucopenia after two injections with adalimumab. Clin Exp Dermatol. 2009;34(8):e1004-1005. doi: 10.1111/j.13652230.2009.03665.x

4. Ottaviani S, Cerf-Payrastre I, Kemiche F, Pertuiset E. Adalimumabinduced neutropenia in a patient with rheumatoid arthritis. Joint Bone Spine. 2009;76(3):312-313.doi: 10.1016/j.jbspin.2008.09.017

5. Bessissow T, Renard M, Hoffman I, Vermeire S, Rutgeerts P, Van Assche G. Review article: non-malignant haematological complications of anti-tumour necrosis factor alpha therapy. Aliment Pharmacol Ther. 2012;36(4):312-323. doi: 10.1111/j.1365-2036.2012.05189.x

6. Azevedo VF, Silva MB, Marinello DK, Santos FD, Silva GB. Leukopenia and thrombocytopenia induced byetanercept: two case reports and literature review. Revista brasileira de reumatologia. 2012;52(1):110112.

7. Bessissow T, Renard M, Hoffman I, Vermeire S, Rutgeerts P, Van Assche G. Review article: non-malignant haematological complications of anti-tumour necrosis factor alpha therapy. Aliment Pharmacol Ther. 2012;36(4):312-323. doi: 10.1111/j.1365-2036.2012.05189.x

8. Rajakulendran S, Gadsby K, Allen D, O’Reilly S, Deighton C. Neutropenia while receiving anti-tumour necrosis factor treatment for rheumatoid arthritis. Ann Rheum Dis. 2006;65(12):1678-1679.

9. Sebastian S, Ashton K, Houston Y, Diggory TM, Dore P. Anti-TNF therapy induced immune neutropenia in Crohns disease- report of 2 cases and review of literature. J Crohns Colitis. 2012;6(6):713716. doi: 10.1016/j.crohns.2012.01.014 